\title{
OPTIC NEUROPATHY IN URAEMIC PATIENTS ON DIALYSIS
}

\author{
SAJJAD HAIDER, NICHOLAS J. ASTBURY and DAVID V. HAMILTON \\ Norwich
}

\begin{abstract}
SUMMARY
Optic nerve disease has been reported in patients with renal failure. A toxic aetiology has been postulated. The incidence of this complication of renal failure has not been determined. We observed 60 patients with renal failure on dialysis for $\mathbf{2 4}$ months to determine the incidence of optic neuropathy. Four patients developed typical acute ischaemic optic neuropathy. Their case notes were reviewed in order to determine the pathogenesis. Each patient had long-standing renal failure and its sequelae of anaemia and hypertension. The immediate causes of ischaemia were hypotension in 1, severe anaemia in 1, and generalised atherosclerosis in 2 patients.
\end{abstract}

Optic neuropathy is a well-known complication of renal failure/dialysis. A neurotoxic type of optic neuropathy in uraemic patients with a serum urea nitrogen level of more than $35.7 \mathrm{mmol} / \mathrm{l}$ was reported by Knox and associates, who implicated hypertension and anaemia as well as a raised serum urea nitrogen. Medical management with haemodialysis was followed by improvement of vision in 4 of their patients. In 1 patient resumption of corticosteroid therapy was followed by improvement in vision, but the patient whose vision improved the most rapidly was managed by prompt use of both dialysis and oral corticosteroids.

Hamad and associates described a similar type of optic neuropathy ${ }^{2}$ in patients maintained on dialysis. In 1 case desferrioxamine toxicity was suspected. Other risk factors identified were again hypertension, anaemia and a raised serum urea nitrogen. Their third patient had profound loss of vision, and the likelihood of an atypical non-arteritic acute ischaemic optic neuropathy (AION) in a young patient in that particular clinical setting was noted.

In contrast, all 4 of our patients had typical non-arteritic acute ischaemic optic neuropathy.

\section{PATIENTS AND METHODS}

All 60 patients attending the renal dialysis unit had oph-

Correspondence to: Sajjad Haider, FCOphth, Registrar, Department of Ophthalmology, West Norwich Hospital, Bowthorpe Road, Norwich NR2 3TU, UK. thalmic screening for optic neuropathy at the outset. This was carried out at the bedside in the renal dialysis unit and comprised measurement of visual acuity using a reduced Snellen test at $38 \mathrm{~cm}$ and examination of the visual fields using the confrontation method ( $3 \mathrm{~mm}$ white and red targets). An afferent pupillary defect was sought and the appearance of the optic disc was noted. Any positive findings were further investigated with a full-sized Snellen chart, refraction and Goldman perimetery. Patients were instructed to report back if they experienced any visual symptoms. On presentation, in addition to a full history and relevant examination, a history and clinical features suggestive of migraine, carotid artery disease, compression or infiltration of the optic nerve were sought in order to rule them out. Temporal arteritis was excluded where necessary by performing a temporal artery biopsy. All patients were reviewed again after 24 months.

\section{RESULTS}

Four patients developed sudden, painless loss of vision with altitudinal or arcuate field loss. They were also noted to have an afferent pupillary defect, a swollen and/or pale optic disc with grossly normal retinal vessels and no emboli. None of them had any premonitary symptoms except for case 2 , who experienced amaurosis fugax. They were all diagnosed as having non-arteritic acute ischaemic optic neuropathy. Cases 1 and 3 had anterior acute ischaemic optic neuropathy whilst cases 2 and 4 had posterior acute ischaemic optic neuropathy. The latter diagnosis was reached by typical clinical features (mode of onset, type of visual fields and progress) and by excluding a compressive aetiology.

\section{CASE REPORTS}

Case 1

A 53-year-old Caucasian man, a non-insulin-dependent diabetic, presented with blurring of vision in his left eye for 1 week. There was no ocular pain or headache. The blurring was rapidly progressive and affected mainly the 
lower half of his visual field. His visual acuity was $6 / 5$ in the right eye and 6/6 in the left eye. A left afferent pupillary defect was noted. His Goldman visual fields are shown in Fig. 1. In addition, colour discrimination was grossly deficient and the left optic disc was pale with blurred superior margins. The retinal vasculature was entirely normal and no emboli were found in the retinal vessels. An erythrocyte sedimentation rate (ESR) performed at the time was $21 \mathrm{~mm} /$ hour. There was no carotid bruit.

Chronic renal failure was secondary to polycystic kidneys that had been diagnosed 25 years previously, and he had received three renal transplants (1975, 1976 and 1978) but was currently on chronic intermittent haemodialysis awaiting a further renal transplant. One week prior to the eye consultation he required admission because of the development of severe hypotension (blood pressure 80/ $40 \mathrm{mmHg}$ ). In 1974 he had first developed hypertension and was at that time treated with beta blockers, but over the past 15 years he had not required treatment. Over the preceding weeks he had, however, suffered a number of brief hypotensive episodes, precipitated during dialysis as a result of marked ultrafiltration to control high interdialysis fluid weight gain. He was severely anaemic secondary to his renal failure and had been treated since November 1990 with erythropoietin. At the time of presentation his haemoglobin level was $9 \mathrm{~g} / \mathrm{dl}$. Since August 1990 he had suffered angina and this was treated with isosorbide and beta blockers. Biochemical tests showed a pre- and post-dialysis urea level varying between $25 \mathrm{mmol} / \mathrm{l}$ and 16 $\mathrm{mmol} / \mathrm{l}$, and a serum creatine level between $1075 \mu \mathrm{mol} / \mathrm{l}$ and $850 \mu \mathrm{mol} / \mathrm{l}$.

\section{Case 2}

A 42-year-old Caucasian woman complained of episodes of blurring of vision in her left eye. Visual acuity was $6 / 9$ in the left eye improving to $6 / 4$ on refraction $(-0.25 /+0.25$ @ 180). There was no afferent pupillary defect. Visual fields were normal. There was no pain on ocular movement. She was reviewed after 3 weeks. There was no

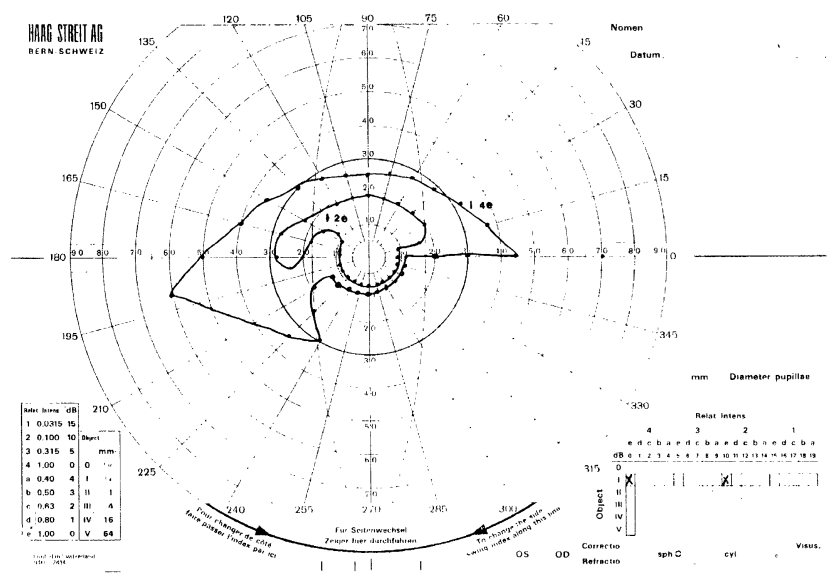

Fig. 1. Case 1: visual field of left eye. With target I $4 e$ : generalised constriction, especially inferiorly; alignment along horizontal meridian nasally (altitudinal field defect). With target I 2 : large arcuate scotoma extending to periphery. change in visual acuity but she complained of visual disturbance in the left eye, similar to the original episodes which had now become constant. She had developed an afferent pupillary defect and an arcuate field defect (Fig. 2). The optic disc showed slight pallor on ophthalmoscopy. Visual evoked potential from the left eye was significantly delayed. A CT scan ruled out a compressive lesion. Her intraocular pressure was normal. There was no abnormality in her other eye. There was no history of migraine. One year later the visual field defect had remained static.

Renal function impairment was first detected in this patient in 1974. An ultrasound scan at that time showed bilaterally small kidneys. Dialysis was commenced in 1988. In February 1989 she had cadaveric renal transplantation. Deterioration in her renal function was detected in February 1990 and since that time there had been a steady deterioration. At the time of presentation with visual complaints she had severe anaemia (haemoglobin $6.6 \mathrm{~g} / \mathrm{dl}$ ) and is now receiving erythropoietin therapy subcutaneously. A renal biopsy in February 1991 showed the presence of chronic rejection. Her blood pressure is currently satisfactory although she has been hypertensive in the past. She has no signs of autonomic neuropathy. Her blood urea level is around $35 \mathrm{mmol} / \mathrm{l}$ and her serum creatine level $990 \mu \mathrm{mol} / \mathrm{l}$.

\section{Case 3}

A 77-year-old Caucasian woman complained of rapid loss of vision in both eyes, the left eye being more severely affected than the right. Visual acuities were recorded as $6 / 60$ right and finger counting at $0.5 \mathrm{~m}$ left. Her visual field is shown in Fig. 3. An afferent pupillary defect was demonstrated in the left eye. The fundi showed a few scattered cotton wool spots but the retinal vessels were of normal calibre and no emboli were visualised. The left optic disc margins were blurred. There were no signs of carotid artery disease and she had no clinical features of temporal arteritis, but the erythrocyte sedimentation rate was found to be raised to $109 \mathrm{~mm} /$ hour. She was started on systemic steroids but a temporal artery biopsy was nega-

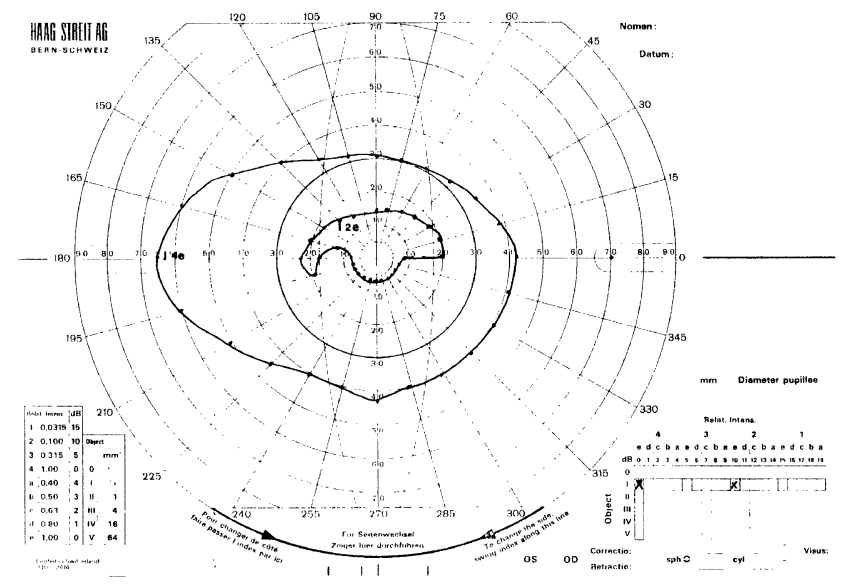

Fig. 2. Case 2: visual field of left eye. With target I 4e: normal periphal field. With target I 2e: inferior arcuate scotoma, 'breaking through' to periphery; nasal altitudinal field defect. 


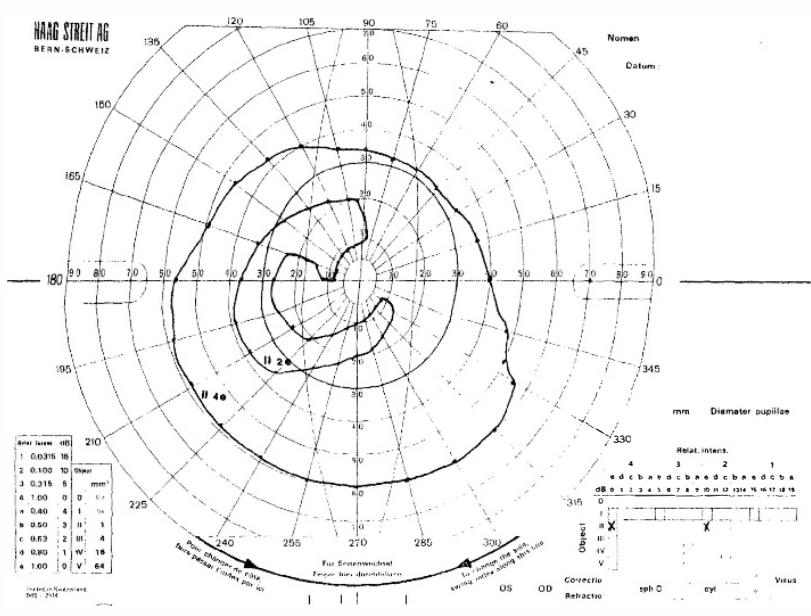

Fig. 3. Case 3: visual field of left eye. With target II 4e: normal peripheral field. With target II $2 e$ : large arcuate defects connected with blind spot, extending to nasal periphery; horizontal alignment just nasal to blind spot.

tive for active giant cell arteritis and the steroids were discontinued.

She was known to have had hypertension since 1974 and more recently (mid 1990) congestive cardiac failure, ischaemic heart disease and anaemia (haemoglobin $7 \mathrm{~g} /$ dl). She had had chronic renal failure for 20 years for which she had been on chronic ambulatory peritoneal dialysis and was recently found to have had shrunken kidneys on ultrasound. She had been an inpatient for the preceding 4 months for management of staphylococcal septicaemia, fluid overload, heart failure and gout. She died from heart failure 3 months after presenting with her ocular complaints. At the time of presentation her urea level was $35 \mathrm{mmol} / \mathrm{l}$ and serum creatinine level 650 $\mu \mathrm{mol} / \mathrm{l}$.

\section{Case 4}

A 32-year-old diabetic Caucasian man who had previously been treated for bilateral proliferative diabetic retinopathy with panretinal photocoagulation, complained of rapid loss of vision in his right eye to counting fingers which improved to $6 / 60$ over the following week. He had previously had stable vision of $6 / 18$ in each eye. On examination he had a right afferent pupillary defect, a colour discrimination defect and a visual acuity of finger counting at $1 \mathrm{~m}$. His visual field is shown in Fig. 4. This deterioration of the visual field was found to be recent after comparison with previous visual fields. The right optic disc was not swollen but pale, the retinal vessels were of normal calibre and no emboli were visualised. There was no history of migraine nor any carotid bruit.

He had been an insulin-dependent diabetic for the previous 20 years, receiving chronic intermittent haemodialysis for the management of chronic renal failure secondary to diabetic nephropathy. His diabetic control had been satisfactory and he had been hypertensive for 4 years, controlled on captopril, nifedipine and atenolol. $\mathrm{He}$ had been anaemic (haemoglobin $9 \mathrm{~g} / \mathrm{dl}$ ) and had recently developed angina. He also had a family history of ischaemic heart disease. A renal transplant failed in 1988 because of an iliac mycotic aneurysm and septicaemia and he required ligation of the femoral artery, femoro-femoral bypass and eventually a transplant nephrectomy. Because of his peripheral vascular disease he was maintained on anticoagulation. Biochemical tests showed a pre- and post-dialysis urea level of $28 \mathrm{mmol} / \mathrm{l}$ and $12 \mathrm{mmol} / \mathrm{l}$ respectively and a serum creatinine level between 1200 and $600 \mu \mathrm{mol} / \mathrm{l}$. The patient died of a massive cerebrovascular accident (cerebral haemorrhage) 5 weeks after presenting with ischaemic optic neuropathy.

\section{DISCUSSION}

Pathogenesis of AION primarily involves interference with the posterior ciliary artery blood supply to the prelaminar optic nerve. ${ }^{3}$ This might be brought about by decreased blood delivery, increased resistance to blood supply or low blood oxygen carrying capacity. ${ }^{4}$ Uraemic patients may often have coexisting pathology such as hypertension, atherosclerosis (increased resistance) and anaemia (low blood oxygen carrying capacity), predisposing them to ischaemic optic neuropathy.

Various authors have documented an association between acute ischaemic optic neuropathy and hypotension, during general surgery, ${ }^{5}$ following cardiopulmonary bypass surgery ${ }^{6}$ and following cardiopulmonary arrest. ${ }^{7}$ Karen et $a l .{ }^{8}$ reported a case of anterior ischaemic optic neuropathy as a result of haemodialysis-associated hypotension (decreased blood delivery). Hypotension is a serious and frequent complication of dialysis.'

Our first patient had multiple episodes of severe hypotension. The temporal relationship between these episodes and the acute ischaemic optic neuropathy suggests that the hypotension was the initiating event. His history illustrates the different factors contributing to the hypotension, which included marked ultrafiltration (necessary to control his weight gain) and the use of beta blockers and isosorbide for angina, combined with autonomic neuropathy associated with his chronic renal failure.

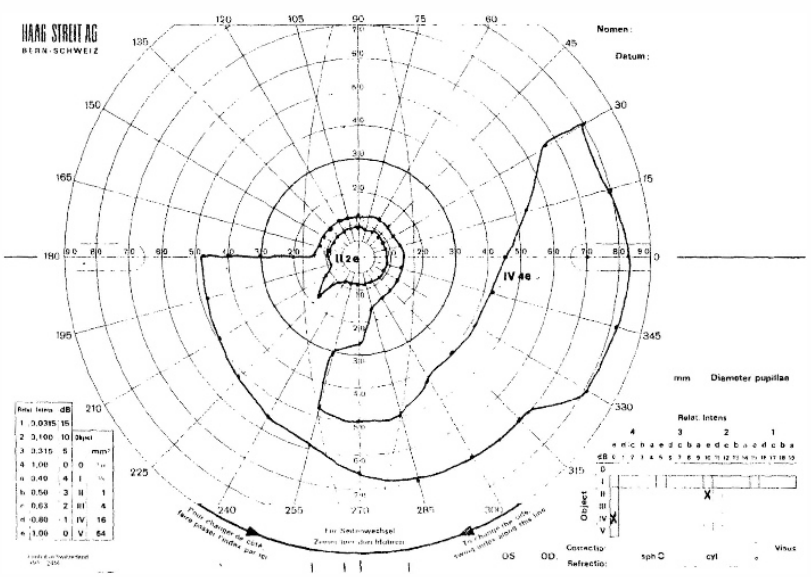

Fig. 4. Case 4: visual field of right eye. With target IV 4e: inferior arcuate scotoma, connected with blind spot and superior arcuate scotoma 'breaking through' to periphery; note horizontal alignment in nasal half of visual field (altitudinal defect). With target II $2 e$ : generalised constriction. 
Anaemia has been implicated before in the aetiology of AION.$^{10}$ Foulds hypothesised that a low haematocrit could sufficiently reduce the blood's oxygen carrying capacity, resulting in $\mathrm{AION}^{4}$ In our second patient episodes of amaurosis fugax were followed by onset of AION. This was preceded by an episode of severe anaemia. Red cell transfusion abolished the amaurosis fugax, but signs of AION developed in the involved eye. In the third patient anaemia again appears to be an important factor.

The third and fourth patients died within 3 months of the onset of acute ischaemic optic neuropathy, one patient from a cerebral haemorrhage and the other from cardiac failure. They both had generalised atherosclerosis (increased resistance to blood supply) in addition to suffering from renal failure. The development of acute ischaemic optic neuropathy in these two patients is likely to be a manifestation of atherosclerosis.

\section{CONCLUSION}

With the expansion of the demand for renal replacement therapy, an increasing number of patients are receiving dialysis, often in the presence of multiple pathology including diabetes, generalised atherosclerosis and hypertension. Optic neuropathy seems to be a common complication of renal failure treated with dialysis and our study indicates that ischaemia rather than toxicity is the causative mechanism responsible for triggering it in these four cases. The cases presented above underline the heterogeneous nature of the factors underlying ischaemia in the clinical setting of renal failure, which included hypotension, anaemia and atherosclerosis.

Key words: Dialysis, Ischaemic optic neuropathy, Uraemia.

\section{REFERENCES}

1. Knox DL, Hanneken AM, Miller NR, Gonzalea WL. Uraemic optic neuropathy. Arch Ophthalmol 1988;106: $50-4$.

2. Hamed LM. Optic neuropathy in uraemia. Am J Ophthalmol 1989;108:30-5.

3. Hayreh SS. Anterior ischaemic optic neuropathy. Br J Ophthalmol 1974;58:955-89.

4. Foulds W. Visual disturbance in systemic disorders: optic neuropathy and systemic disease. Trans Opthalmolol Soc UK 1969;89:125-46.

5. Rizzo J, Simmons L. Posterior ischaemic optic neuropathy during general surgery. Am J Ophthalmol 1987;103: 808-11.

6. Sweeney PJ et al. Ischaemic optic neuropathy: a complication of cardiopulmonary bypass surgery. Neurology 1982; 32:560-2.

7. Sundaram MBM, Avram D, Cziffer A. Unilateral ischaemic optic neuropathy following systemic hypotension. J R Soc Med 1986;79:250.

8. Karen S, Servilla MD, Grogge GC. Anterior ischaemic optic neuropathy as a result of haemodialysis associated hypotension. Am J Kidney Dis 1986;8(1):61-3.

9. Henderson LW. Symptomatic hypotension during haemodialysis. Kidney Int 1980;17:571-6.

10. Golnik KC, Newman SA. Anterior ischaemic optic neuropathy associated with macrocytic anaemia. J Clin Neuro Ophthalmol 1990;10:244-7. 